\title{
Recomendação de Conteúdo em Ambientes de Aprendizagem Baseados em Questionamento
}

\author{
Otávio Costa Acosta \\ Universidade Federal do Rio Grande do Sul - ocacosta@inf.ufrgs.br \\ Eliseo Berni Reategui \\ Universidade Federal do Rio Grande do Sul - eliseoreategui@gmail.com
}

\begin{abstract}
Resumo: O presente trabalho apresenta um modelo de um ambiente virtual de aprendizagem baseado em questionamentos que dá suporte aos alunos através de funções de recomendação de conteúdo. Este processo de aprendizagem pode ser utilizado como forma de engajar os estudantes ativamente na investigação e descoberta de temas de estudo. As recomendações de conteúdo são baseadas em um texto selecionado pelo professor que determina o tema de uma atividade. Estas recomendações podem ser desde textos que auxiliem na elaboração de questionamentos, imagens, vídeos ou até autores que escrevem sobre tema proposto. Esta abordagem amplia o funcionamento da ferramenta SMILE, ferramenta para apoio à aprendizagem baseada em questionamento desenvolvida na Universidade de Stanford.
\end{abstract}

Palavras-Chave: aprendizagem baseada em questionamento, ambientes virtuais de aprendizagem, sistemas de recomendação.

\begin{abstract}
This paper presents an inquiry-based learning environment that supports students through content recommendation functions. This learning process may be used as a way of engage students actively in research and discovery. The content recommendations are based on a text selected by the teacher, who determines the subject of an activity. These recommendations can be texts that assist the students in making questions, as well as images, videos or authors who write about the proposed topic. This approach extends the tool SMILE, an inquiry-based learning tool developed at Stanford University.
\end{abstract}

Keywords: inquiry-based learning, virtual learning environments, recommender systems.

\section{Introdução}

A aprendizagem baseada em questionamento, do inglês inquiry-based learning, é definida como uma abordagem de aprendizagem guiada pelo processo de elaboração de perguntas pelos próprios aprendizes (Barret et al., 2005). Nesta abordagem, busca-se instigar os estudantes à elaboração de questões sobre determinado tema, como forma de levá-los a desenvolver suas próprias investigações sobre o assunto.

A abordagem, com origem na década de 60 , surgiu como resposta às limitações dos métodos tradicionais de ensino nos quais os alunos eram obrigados a memorizar fatos disponíveis nos materiais escolares (Bruner, 1961). Hoje, a aprendizagem baseada em questionamento é vista como uma forma de aprendizagem ativa, onde a construção 
do conhecimento se dá por meio do desenvolvimento de atividades experimentais e analíticas (Seol et al., 2011). No que diz respeito às práticas pedagógicas fundamentadas nesta abordagem, a aprendizagem baseada em questionamento possibilita que os estudantes trabalhem individualmente ou em grupo na resolução de problemas elaborados por eles mesmos, deixando ao professor o papel de mediador deste processo.

Muitos trabalhos na área da tecnologia na Educação tem-se apoiado na abordagem de aprendizagem baseada em questionamento. Por exemplo, Yoder (2003) apresenta casos de construção de webquests fundamentadas na aprendizagem baseada em questionamento, Mott e Lester (2006) descrevem um ambiente de aprendizagem centrado no desenvolvimento de narrativas e ancorado na aprendizagem baseada em questionamento. Já Lim (2004) discute questões e problemas relacionados ao emprego da abordagem na web, um ambiente considerado amplo, pouco estruturado e complexo.

Outro trabalho relacionado à aprendizagem baseada em questionamento mediada pela tecnologia é o projeto SMILE (Stanford Mobile Inquiry-based Learning Environment), cujo objetivo principal é possibilitar aos alunos a criação e compartilhamento de questões relacionadas a um determinado assunto (Kim et al., 2011). Uma ferramenta para gerenciamento das perguntas e respostas elaboradas pelos estudantes foi desenvolvida no projeto, com foco no emprego de dispositivos móveis. Resultados em diversos experimentos com o uso da ferramenta demonstraram o envolvimento e bom desempenho dos alunos nas atividades propostas.

A pesquisa aqui apresentada tem como foco a ampliação da ferramenta SMILE por meio da integração de um sistema de recomendação de conteúdo a esta, com o objetivo de facilitar os processos investigativos e ampliar a reflexão dos estudantes com relação aos temas abordados. A próxima seção apresenta a ferramenta SMILE de forma mais detalhada, buscando descrevê-la com relação a aspectos tanto técnicos quanto pedagógicos. A seção 3 apresenta os sistemas de recomendação, focando nas técnicas de recomendação de conteúdo selecionadas para utilização junto à ferramenta SMILE. A seção 4 descreve o sistema desenvolvido, apresentando sua arquitetura geral e telas de funcionamento. A seção 5 apresenta considerações finais bem como direcionamentos para trabalhos futuros.

\section{Ferramenta SMILE}

A ferramenta SMILE foi desenvolvida com o objetivo de facilitar o desenvolvimento de atividades de aprendizagem baseada em questionamento por meio do uso de dispositivos móveis. Entende-se que as perguntas criadas pelos próprios alunos, de acordo com esta abordagem, possam desempenhar um papel importante no processo de aprendizagem na medida em que levam os alunos ao questionamento, à reflexão e à resolução dos próprios questionamentos. Ao se questionarem sobre determinado tema, os alunos acabam delineando suas próprias investigações, consultam materiais diversos e assim criam novas associações com conhecimentos anteriores (Commeyras, 1995). Esta prática está alinhada às teorias construtivistas que colocam o aluno como sujeito ativo nos processos de aprendizagem (Piaget, 1977).

A ferramenta SMILE implementa um sistema de gerenciamento de perguntas e respostas criadas e compartilhadas pelos estudantes. Basicamente, a ferramenta é composta por duas aplicações distintas:

- Junction Quiz - destinada a alunos, possibilita a edição de questões e elaboração de respostas para estas. A aplicação, desenvolvida para dispositivos móveis, 
envia as questões e respostas elaboradas para um servidor central, possibilitando que o professor e outros alunos as consultem.

- Junction Quiz Controller - destinada a professores, possibilita a visualização e gestão das questões e respostas elaboradas pelos alunos.

Para que seja possível a comunicação e o correto funcionamento entre as aplicações citadas, é necessário que os equipamentos utilizados pelas mesmas estejam conectados através de uma rede local. Caso estes dispositivos possuam recursos de conexão em rede sem fio, uma rede local pode ser facilmente implantada a partir da utilização de um roteador de rede sem fio.

A Figura 1 apresenta duas telas da ferramenta Junction Quiz, que permite aos alunos compartilhar com seus colegas as questões. A ferramenta também permite a inserção de imagens que possam auxiliar a compreensão das questões e respostas desenvolvidas.
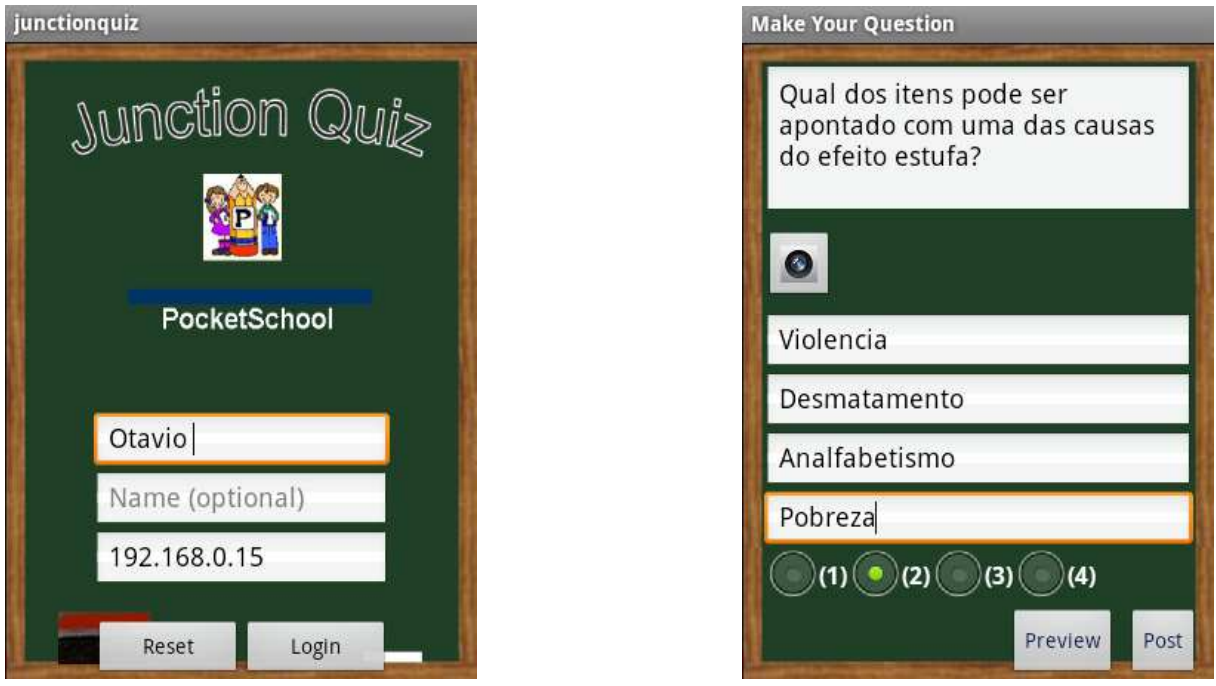

Figura 1: Tela de abertura da ferramenta Junction Quiz e tela de elaboração de perguntas

Já a ferramenta Junction Quiz Controller permite ao professor acompanhar, em tempo real, as perguntas e respostas elaboradas pelos alunos. Também é possível salvar as perguntas realizadas para posterior uso em uma nova atividade.

Contudo, nenhuma das ferramentas possui suporte para a busca por conteúdos na internet, como forma de apoiar os alunos em suas investigações. A próxima seção apresenta os sistemas de recomendação como uma proposta de abordagem para facilitar aos alunos a busca por diferentes materiais que possam levá-los à resolução de seus questionamentos.

\section{Sistemas de Recomendação}

Com o contínuo aumento da inclusão digital e a consequente facilidade ao acesso a grandes quantidades de informações disponíveis na web, torna-se cada vez mais difícil a tarefa de escolha de conteúdos dentre as diversas opções disponíveis. Os sistemas de recomendação foram desenvolvidos como forma de auxiliar os usuários na identificação de itens de seu interesse (Resnick e Varian, 1997). Tipicamente, busca-se identificar perfis de interesse dos usuários a partir dos itens por eles avaliados. Um dos grandes desafios deste tipo de sistema é realizar a combinação adequada entre as 
expectativas dos usuários e os documentos, serviços ou pessoas a serem recomendados aos mesmos, ou seja, definir e descobrir estes relacionamentos (Cazella et al., 2010).

A seguir, serão descritas algumas das técnicas de recomendação mais frequentes, sendo elas: Filtragem Baseada em Conteúdo, Filtragem Colaborativa e Filtragem Híbrida.

\subsection{Filtragem Baseada em Conteúdo}

A abordagem baseada em conteúdo teve origem na área de recuperação de informação, focando principalmente na recomendação de itens com informações textuais, como documentos e web sites. As melhorias sobre os sistemas tradicionais de recuperação de informação vieram com a utilização do perfil do usuário, que contém suas preferências e necessidades. As informações sobre o perfil do usuário podem ser obtidas pelo próprio usuário, como uma consulta realizada por ele ou coletadas através do conteúdo dos itens que o usuário utiliza. Uma técnica muito comum neste tipo de abordagem é a indexação de frequência de termos. Neste tipo de indexação, informações dos documentos e necessidades dos usuários são descritas por vetores com uma dimensão para cada palavra que ocorre na base de dados. Cada componente do vetor é a frequência que a respectiva palavra ocorre em um documento ou na consulta do usuário. Sendo assim, os vetores dos documentos que estão próximos aos vetores de consulta são considerados os mais relevantes para ele.

Desta forma, a filtragem baseada em conteúdo parte do princípio de que os usuários tendem a interessar-se por itens similares aos que demonstraram interesse no passado, definindo então, a similaridade entre os itens (Herlocker, 2000). Em alguns casos, pode haver maior dificuldade para estabelecer esta similaridade, como por exemplo, para que seja estabelecida a similaridade entre itens como roupas e brinquedos, talvez seja necessária a identificação dos atributos nos itens a serem comparados (peso, preço ou marca). No caso dos itens serem documentos textuais, este processo de comparação pode ser facilitado, pois documentos podem ser considerados similares se compartilharem termos em comum. Sendo assim, a filtragem baseada em conteúdo é bastante adequada à recomendação de itens textuais, onde o conteúdo é geralmente associado a palavras-chave (Salton and McGill, 1983).

\subsection{Filtragem Colaborativa}

A Filtragem Colaborativa se diferencia da filtragem baseada em conteúdo exatamente por não exigir a compreensão ou reconhecimento do conteúdo dos itens, atendendo pontos que ficaram em aberto na abordagem baseada em conteúdo (Ansari et al. 2000).

Nos sistemas colaborativos a essência está na troca de experiências entre as pessoas que possuem interesses comuns e os itens são filtrados baseados nas avaliações feitas pelos usuários. Segundo Herlocker (2000), os primeiros sistemas de filtragem colaborativa requeriam usuários para especificar o relacionamento de predição entre suas opiniões, ou de modo explícito indicar os itens de interesse. Porém, em seguida estes sistemas automatizaram todo o procedimento através da coleção das pontuações dos itens pelos usuários. Um usuário de um sistema de filtragem colaborativa deve, portanto, pontuar cada item experimentado, indicando o quanto este item casa com sua necessidade de informação. Estas pontuações são coletadas para grupos de pessoas, permitindo que cada usuário se beneficie das pontuações (experiências) apresentadas por outros usuários na comunidade. Sistemas de filtragem colaborativa simples apresentam para o usuário uma média de pontuações para cada item com potencial de 
interesse. Esta pontuação permite ao usuário descobrir itens que são considerados de interesse pelo grupo e evitar os itens que são considerados de pouco interesse. Sistemas mais avançados descobrem de maneira automática relações entre usuários (vizinhos mais próximos), baseado na descoberta de padrões comuns de comportamento.

A filtragem colaborativa apresenta algumas vantagens, como por exemplo, a possibilidade de apresentar aos usuários recomendações inesperadas, ou seja, o usuário poderia receber recomendações de itens que não estavam sendo pesquisados de forma ativa. Outra contribuição importante dos sistemas de filtragem colaborativa se refere à possibilidade de formação de comunidades de usuários pela identificação de seus gostos e interesses similares.

\subsection{Filtragem Híbrida}

A Filtragem Híbrida emprega técnicas advindas tanto da abordagem de Filtragem Colaborativa, quando da Filtragem baseada em Conteúdo. A ideia é baseada na busca de itens de acordo com os perfis de interesse dos usuários, mas considerando também a similaridade de conteúdo entre os itens. Ao integrar ambas as técnicas, a Filtragem Híbrida busca incorporar as vantagens tanto da Filtragem Colaborativa quanto da Filtragem baseada em Conteúdo, ao mesmo tempo em que minimiza possíveis limitações de uma ou outra abordagem (Herlocker, 2000).

No que diz respeito à abordagem adotada para recomendação de conteúdo na plataforma SMILE, optou-se pela Filtragem baseada em Conteúdo. A próxima seção descreve o sistema desenvolvido e argumenta sobre esta decisão técnica.

\section{Um Ambiente de Aprendizagem Baseado em Questionamento com Recomendação de Conteúdo}

A partir da ideia apresentada no projeto SMILE e na abordagem de recomendação de conteúdo por sistemas de recomendação, este artigo apresenta a remodelagem da ferramenta Junction Quiz, agregando funções de recomendação que podem apoiar alunos na busca por materiais para auxiliá-los em suas investigações. A ferramenta desenvolvida foi chamada de Rec Quiz, fazendo a alusão da recomendação de conteúdos associada à ferramenta Junction Quiz.

Lane (2007) apresenta diferentes possibilidades de práticas pedagógicas com base na aprendizagem baseada em questionamento. Algumas destas abordagens podem ser mais guiadas, com o professor cumprindo um papel de acompanhamento e direção das investigações feitas pelos estudantes. Outras abordagens são mais livres, dando aos estudantes mais liberdade na condução de suas próprias investigações. Aqui, a abordagem pedagógica que empregamos é uma variação daquela classificada por Bonstetter (1998) como Investigação Dirigida pelo Estudante. Nesta, o estudante é responsável por toda a pesquisa sobre o tema proposto pelo professor, com a intervenção deste somente para adequação das questões elaboradas. A variante que propomos é a introdução do tema pelo professor por meio de um texto disparador - cujo objetivo é apresentar o tema aos alunos e instigá-los a investigar e realizar suas próprias descobertas sobre o assunto.

Ao iniciar um projeto de aprendizagem baseada em questionamento, o professor apenas informa à ferramenta Junction Quiz Controller o texto que deverá ser utilizado como texto disparador do projeto. Em seguida, os alunos podem se logar na ferramenta Junction Quiz e assim acessar o texto disponibilizado, como forma de realizar uma leitura inicial sobre o tema a ser trabalhado. A ideia deste texto é que seja relativamente 
6

curto, com informações básicas para introdução do assunto e possivelmente algumas questões que instiguem os estudantes a elaborar novas perguntas e pesquisarem sozinhos suas respostas.

Os estudantes começam então a seção de elaboração de questões sobre o tema. $\mathrm{Na}$ medida em que criam suas questões e procuram descrever as respostas usando o editor da ferramenta Rec Quiz, o sistema emprega um minerador de textos para extrair termos relevantes das produções dos alunos (Reategui et al., 2011) e montar uma busca na web a partir destas. Os materiais retornados nesta busca (textos, imagens, vídeos e outros) são então filtrados e recomendados aos alunos, como forma de ampliar as buscas feitas e também os tipos de materiais empregados na construção das investigações. A Figura 2 mostra o diagrama que representa este processo.

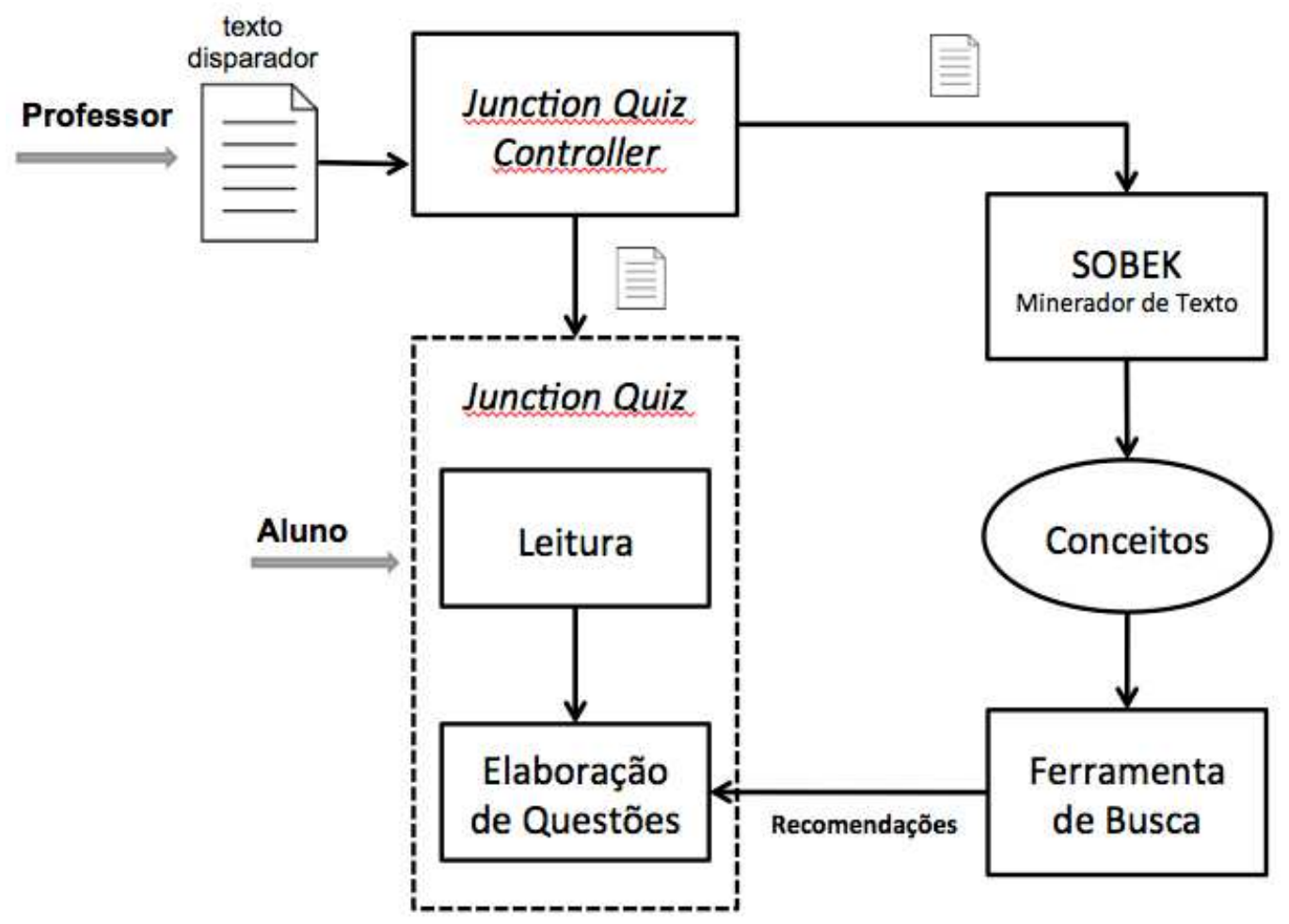

Figura 2: Diagrama do Sistema Rec Quiz

Além de recomendar aos alunos os materiais advindos da web, o sistema de recomendação desenvolvido monitora os materiais realmente acessados e utilizados pelos alunos, com o objetivo de avaliar o uso das recomendações feitas e empregar tal avaliação em novas recomendações. Estas podem incluir, além de textos, imagens e vídeos, autores e especialistas no tema proposto pela atividade (Loh et al., 2011). A Figura 3 mostra uma tela do sistema, visualizada em um tablet, na qual o aluno elabora uma questão sobre o efeito estufa e recebe recomendação de imagens e documentos relacionados à pergunta elaborada. 


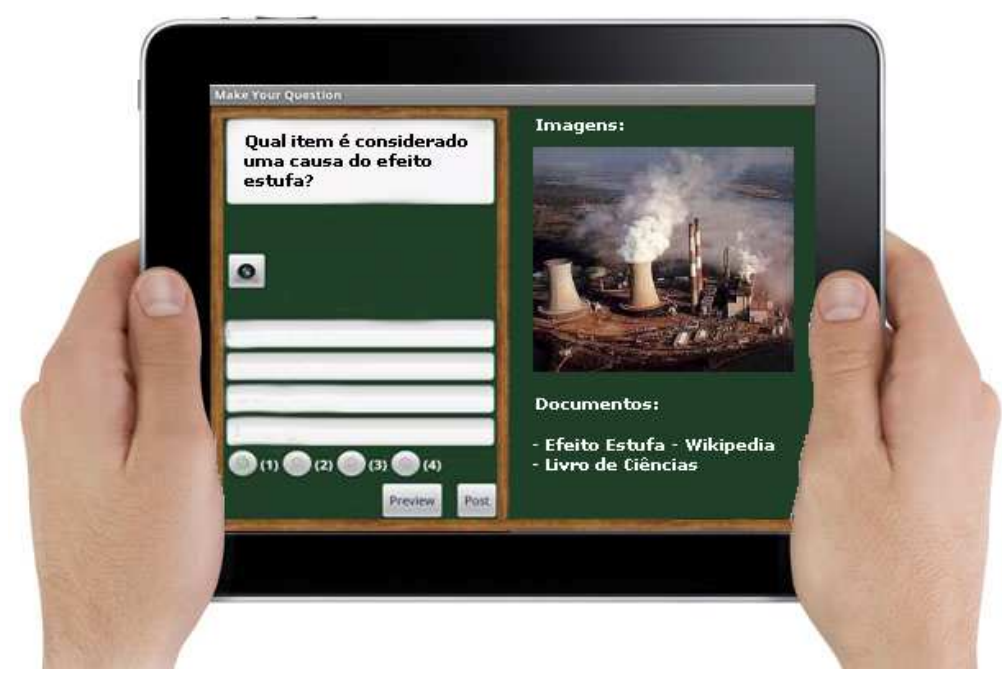

Figura 3: Sistema Rec Quiz empregado na elaboração de pergunta sobre o Efeito Estufa

No que diz respeito à técnica para recomendação escolhida para a aplicação, optou-se pela filtragem de conteúdo pela natureza deste tipo de recomendação. Nesta abordagem, o foco da recomendação está na filtragem de materiais que atendam determinados critérios relacionados ao conteúdo de interesse do usuário. Esta era exatamente a necessidade do sistema aqui proposto. Já a filtragem colaborativa tem como foco identificar interesses comuns em grupos de usuários - o que também poderia ser interessante, mas não essencial no desenvolvimento do Rec Quiz. Já a filtragem híbrida, a qual agrupa características de ambas, poderia ser uma opção também adequada. Contudo, para que realmente pudesse fazer sentido, seria necessário um grupo bastante grande de usuários - o que na prática não ocorre quando imagina-se o sistema sendo empregado no contexto de uma sala de aula. Neste universo, o simples registro da "popularidade" dos itens consultados pelos alunos poderia dar subsídios suficientes para o refinamento das recomendações do sistema.

\section{Considerações Finais}

Este artigo apresentou uma ferramenta para apoio à aprendizagem baseada em questionamento, o qual tem como principal característica a capacidade de realizar recomendações de conteúdos aos alunos relacionados ao tema proposto pelo professor. A ferramenta proposta amplia o funcionamento da ferramenta SMILE, desenvolvida na Universidade de Stanford com o foco em aplicações educacionais para dispositivos móveis.

Com relação à aprendizagem baseada em questionamento, esta é uma abordagem que pode ser utilizada como forma de engajar os estudantes ativamente na investigação e descoberta de temas de estudo comumente elencados nas disciplinas curriculares correntes. Diversos estudos têm demonstrado a eficácia de tal abordagem no que diz respeito ao envolvimento e desempenho dos estudantes na realização das atividades propostas (Seol et al., 2011). No projeto aqui descrito, procurou-se ampliar as funcionalidades da ferramenta SMILE para apoio à aprendizagem baseada em questionamento, integrando-se a ela recomendações de materiais relacionados às questões elaboradas pelos estudantes.

O sistema descrito neste artigo será em breve empregado em testes preliminares no contexto escolar, como forma de avaliar sua real utilização em sala de aula. $\mathrm{O}$ foco 
8

de sua avaliação será agora não apenas no envolvimento dos estudantes, mas também na forma como estes conduzem sua pesquisa ao integrar a ela conteúdos recomendados pelo ambiente Rec Quiz.

\section{Referências Bibliográficas}

ANSARI, A., ESSEGAIER, S., KOHLI, R. Internet Recommendation Systems. Journal of Marketing Research, 2000, v.37, n.3, p. 363-375.

BARRET, T., MAC LABHRAINN, I., FALLON, H. Handbook of Enquiry \& Problem Based Learning. Galway: CELT, 2005. Distribuído sob licença Creative Commons. Disponível em: http://www.nuigalway.ie/celt/pblbook/. Acessado em Maio de 2012.

BONSTETTER, R.J. Inquiry: Learning from the past with an eye on the future. Electronic Journal of Science Education, vol 3, no 1, 1998.

BRUNER, J. S. The act of discovery. Harvard Educational Review, vol 31, no1, 1961. p. 21-32.

CAZELLA, S., NUNES, M. A., REATEGUI, E. (2010) A Ciência do Palpite: Estado da Arte em Sistemas de Recomendação Jornada de Atualização de Informática-JAI 2010CSBC2010, 2010. ed. Rio de Janeiro : Puc RIO, 2010, v.1, p. 161-216.

COMMEYRAS, M., What can we learn from students' questions? Theory into Practice, Vol. 34, Issue 2, 101-106, 1995.

HERLOCKER, J. L. Understanding and Improving Automated Collaborative Filtering Systems, Tese de Doutorado (Doutorado em Ciência da Computação), University of Minnesota, Minnesota, 2000.

KIM, P., GOYAL, A., SEOL, S., DODSON, B., LAM, M. PocketSchool Interactive Learning Ad-hoc Network. To appear in the proceedings of IEEE International Conference on e-Education, Entertainment and e-Management, 2011

LANE, J. Inquiry-Based Learning. The Schreyer Institute for Teaching Excellence, Penn State University, 2007. Disponível em: www.schreyerinstitute.psu.edu/pdf/ibl.pdf . Acessado em Maio de 2012.

LIM, B. Challenges and Issues in Designing Inquiry on the Web. British Journal of Educational Technolog, 2004, Vol 35, No 5, 2004. pp. 627-643.

LOH, S.; LICHTNOW, D.; KAMPFF, A. J.; OLIVEIRA, J. P. M. d. Recommendation of Complementary Material during Chat Discussions. Knowledge Management, [S.1.], v.2, n.4, p.385-399, 2010.

MOTT, B. W., LESTER, J. C. Narrative-Centered Tutorial Planning for Inquiry-Based Learning Environments. Proceedings of the Eighth International Conference on Intelligent Tutoring Systems, vol. 4053, Berlin: Springer, 2006. pp. 675-684

PIAGET, J., The Role of Action in the Development of Thinking. In W. F. Overton, \& J. M. Gallagher," Advances in Research and Theory. New York: Plenum Press, 1977.

REATEGUI, E., EPSTEIN, D., LORENZATTI, A., KLEMANN, M. Sobek: a Text Mining Tool for Educational Applications. Em Anais do International Conference on Data Mining, Las Vegas, Estados Unidos, 2011. p. 59-64. 
9

RESNICK, P., VARIAN, H. R. Recommender Systems. Communications of the ACM, New York, 1997, v.40, n.3, pp. 55-58.

SALTON, G., MCGILL J. M. Introduction to Modern Information Retrieval. McGraw Hill, New York, USA, 1983

SEOL, S., SHARP, A., KIM, P. Stanford Mobile Inquiry-based Learning Environment (SMILE): using mobile phones to promote student inquires in the elementary classroom. Proceedings of IEEE World Congress in Computer Science, Computer Engineering, and Applied Computing, 2011

YODER, M. Inquiry Based Learning Using the Internet: research, resources, WebQuests. In 19th Annual Conference on Distance Teaching and Learning, 2005 